\title{
RECEPCIÓN Y ASIMILACIÓN DE LAS TECNOLOGÍAS EN CENTROS ESCOLARES: EL PROYECTO «EL RINCÓN DEL RATÓN» ${ }^{1}$
}

\author{
(RECEPTION AND ASSIMILATION OF TECHNOLOGIES IN SCHOOLS: THE \\ PROJECT «THE MOUSE CORNER»)
}

Ángel San Martín Alonso

Universitat de València

Jorge Martín Domínguez y Elena Ramírez Orellana

Universidad de Salamanca

DOI: $10.5944 / e d u c X X 1.16470$

Cómo referenciar este artículo/How to reference this article:

San Martín, A., Martín, J. y Ramírez, E. (2016). Recepción y asimilación de las tecnologías en centros escolares: El proyecto «El rincón del ratón». Educación XX1, 19(2) 337-358, doi: 10.5944/ educXX1.16470

San Martín, A., Martín, J., and Ramírez, E. (2016). Recepción y asimilación de las tecnologías en centros escolares: El proyecto «El rincón del ratón» [Reception and assimilation of technologies in schools: the project «The mouse corner»]. Educación XX1, 19(2) 337-358, doi: 10.5944/ educXX1.16470

\section{RESUMEN}

En este trabajo indagamos sobre las evidencias en torno a cómo se reciben en los centros escolares los equipamientos tecnológicos (el KidSmart en nuestro caso). Asumimos, a modo de hipótesis, que el grado de asimilación por parte de los miembros de la organización de la tecnología transferida, es un factor determinante del grado de uso. El trabajo de campo se realiza en los centros de educación infantil que participan en el proyecto «El Rincón del Ratón». Mediante grabaciones de clase, encuestas y observaciones hemos tratado de obtener evidencias sobre el problema de investigación. Los primeros resultados ponen de manifiesto el importante, aunque insuficiente, trabajo preparatorio de la transferencia, dificultando la apropiación por parte de los actores y de la organización. Concluimos insistiendo en la necesidad de redefinir el diseño e implementación de los programas de transferencia de tecnología a las organizaciones escolares.

\section{PALABRAS CLAVE}

Transferencia de tecnología; tecnología educativa; educación infantil; administración educativa; cambio tecnológico. 


\section{ABSTRACT}

In this study we inquire into the evidence of how technological equipment (KidSmart in our case) is received in schools. As a hypothesis, we assume that the degree of assimilation by the organization members of the technology transferred is a determining factor in the degree of use. The fieldwork is done in preschools participating in the project «The mouse corner». Through class recordings, surveys and observations we have sought to obtain evidence about the research problem. Early results show the important, albeit insufficient, preparatory work for the transfer, hindering the appropriation by part of the players and the organization. We conclude by stressing the need to redefine the design and implementation of technology transfer programs for school organizations.

\section{KEY WORDS}

Technology transfer; educational technology; preschool; educational administration; technological change.

\section{INTRODUCCIÓN}

Más allá de la consideración que merezca o de los beneficios aportados a la enseñanza, lo cierto es que los centros escolares han mejorado notablemente su equipamiento tecnológico durante estas últimas décadas. Fruto de la iniciativa pública y de la privada, sin demasiada coordinación entre sí, han mantenido diferentes programas de dotación con el propósito de mejorar la «calidad» de la enseñanza. No parece, sin embargo, que los resultados se correspondan demasiado con el esfuerzo inversor, al menos en magnitudes equiparables (Lanier, 2011a). Hecho que suscita no pocas preguntas, si bien en este trabajo nos centraremos en cómo se articula y recibe uno de esos programas de dotación a los centros escolares: «El Rincón del ratón».

Una perspectiva de análisis del equipamiento tecnológico de las organizaciones productivas es la que lo concibe como transferencia de tecnología (TT en lo sucesivo). En la sociedad actual estos procesos son estratégicos, por cuanto los sistemas de innovación y desarrollo producen inventos que luego se han de trasladar como equipamiento a las diferentes instancias y organizaciones. De modo que la expresión TT alude al «proceso de transmisión del saber hacer (know-how, savoir faire), de conocimientos científicos y/o tecnológicos y de tecnología de una organización a otra» (Jiménez, 2003, p. 1). El proceso afecta por igual a los artefactos tanto materiales como inmateriales, organización del trabajo o simbólicos, convirtiendo la transferencia en una especie de conocimiento aplicado en contextos distintos. 
Dado nuestro ámbito de referencia cabe preguntarse: ¿Lo que sucede con el equipamiento de los centros escolares puede interpretarse y estudiarse desde el modelo de TT? Con ciertas salvedades entendemos que sí, en la medida que las acciones complejas que constituyen los programas de TT siguen protocolos muy elaborados. La cuestión está en si con las organizaciones escolares, en tanto que receptoras del equipamiento, se sigue o no alguno de esos protocolos. En este sentido lo primero que llama la atención es que respecto a estas organizaciones, al contrario que sucede en otros ámbitos productivos, no hay demasiados estudios sobre la incorporación de los medios desde la perspectiva de la TT. Circunstancia que contrasta con la abundante literatura a propósito de la cantidad, de las actitudes o de la intensidad de uso de estos equipamientos por parte de estudiantes y docentes.

En los programas de TT intervienen básicamente tres actores: el sistema de innovación tecnológica, los proveedores o difusores y los receptores. Nosotros nos centraremos en el tercer actor, en los centros en tanto que receptores, focalizando la atención sobre cómo se prepara y recibe el equipamiento tecnológico en estas organizaciones. Y lo hacemos con un doble propósito: explorar vías complementarias en la comprensión del tipo de uso que se hace de las TIC en los centros y, vinculado a lo anterior, si en las instituciones escolares se observa lo que mantienen la mayoría de los trabajos sobre la TT respecto al papel crucial que juega la preparación de la entidad para asimilar la tecnología recibida. De manera que: ¿Cómo y mediante qué estrategias se prepara la organización de los centros cuando éstos participan en programas de TT?

\section{ENTRAMADO DEL PROBLEMA DE ESTUDIO}

Como señalan numerosos autores, la sociedad de nuestros días se organiza sobre los principios de un capitalismo reestructurado en torno a la «economía del conocimiento». Modelo en el que la diseminación y redistribución de los hallazgos científico-tecnológicos, son fundamentales para el mantenimiento del mismo. Como es lógico, la institución escolar no escapa a las vicisitudes de este nuevo orden socioeconómico. De hecho, una consigna en tal sentido se formula en el documento programático del Gobierno de España y con el que se da sustento al Plan Escuela 2.0. En él, siguiendo las pautas de la OCDE, se plantea que la «economía de la innovación pivota sobre un núcleo central, que es el conocimiento y su transferencia, desde aquellos puntos en los que se genera» (p. 25) ${ }^{2}$.

Durante las últimas décadas la institución escolar, pese a las críticas frecuentes, no ha dejado de participar en los diferentes programas de TT (Proyecto Atenea y Mercurio, Aldea Digital, InfoXXI, Internet en el Aula, Es- 
cuela2.0), aunque no se hayan reconocido ni gestionado como tales. Llama así la atención que, contrariamente a lo que sucede en los ámbitos no pedagógicos, el grado de equipamiento no se corresponde con las expectativas albergadas. Sí mejora la motivación de los estudiantes, pero existen dudas razonables sobre si el aumento de la dotación mejora o dificulta la coordinación organizativa interna y externa de los centros, si mejoran o dificultan las condiciones de trabajo de los docentes (EACEA-Eurydice, 2011). Por ello es fundamental indagar sobre las razones de semejante fenómeno y, en particular, sobre las circunstancias que concurren en los centros receptores para que disponiendo de los medios no incrementen significativamente su rendimiento (Mominó et al. 2008). Circunstancia en la que tanto el modelo de integración tecnológica que se maneja y el diseño de su estudio parecen relevantes (Liu, Cleborne y LaMont, 2008), así como el papel de las administraciones al regular la relación entre lo público y lo privado en estos programas que son de diseminación de la innovación tecnológica (Onken, Fisher y Li, 2005). En definitiva, ¿cómo se prepara a la organización y sus miembros para que asimilen la tecnología recibida?

\section{Itinerario de los medios hasta los centros escolares}

Los programas de TT incorporan todo tipo de tecnología generada por cualquiera de los agentes integrados en el sistema tecnocientífico, responsables de los diseños y desarrollos de la innovación. En la actualidad, lo que se transfiere de una organización a otra es el know-how pero que, en realidad, este intangible engloba bienes y actividades tan diferentes como: proyectos de $\mathrm{I}+\mathrm{D}+\mathrm{i}$, equipamiento, servicios, asistencia técnica, producción de bienes protegidos, explotación y desarrollo de patentes, propiedad intelectual, movilidad de personal, etc. La innovación y, por tanto, la TT se desarrolla simultáneamente en el sector industrial y en el de servicios, pudiendo afectar a: productos, procesos, posición del producto o servicio en el contexto y el paradigma cuando una organización cambia el modelo de producción del bien (COTEC, 2011, 117 y ss.).

En los programas de TT se pueden diferenciar, en términos descriptivos, dos planos: el cómo y qué tecnología se transfiere (el KidSmart, por ejemplo) y, en segundo lugar, la naturaleza de las estrategias mediante las cuales se acompaña la incorporación de las tecnologías en la organización receptora. Ambos planos son fundamentales, si bien en este trabajo nos centraremos en el segundo por considerar que la iniciativa «El rincón del ratón» presenta numerosas vertientes de análisis sobre el particular. Opción que no debe ocultarnos el cruce de relaciones de determinación que se da entre los dos planos, máxime cuando confluyen sobre un sistema como el escolar que es subsidiario, también en lo que respecta a la TT. De modo que 
no debemos ignorar una cuestión: ¿Qué intereses y propósitos vertebran la TT sobre los sistemas escolares?

No es ningún secreto que la inmensa mayoría de los programas de transferencia responden a intereses y motivaciones extrínsecas a las organizaciones escolares. No atienden tanto a demandas estratégicas de éstas, como a planteamientos políticos o económicos en torno a la «modernización» de la escuela, "aminorar» la brecha entre las aulas y la sociedad, «cambiar» sus prácticas decimonónicas o simplemente manifestación de la «responsabilidad social» de las grandes corporaciones. Bajo estas circunstancias, la comunidad escolar no sólo no se siente interpelada sino que además recela de los cambios sustantivos inducidos en la organización del trabajo por parte de las tecnologías digitales y la cultura asociada a éstas (Bélanger y Thuderoz, 2010; Castells, 2001).

En un contexto tan condicionado por criterios comerciales, resultan razonables las reticencias de una buena parte de los actores escolares hacia algunas iniciativas de esta naturaleza. Destacamos, a este respecto, dos fundamentadas llamadas de atención. Desde la perspectiva comparada y con datos de la OCDE, Pedró (2011, p. 22), afirma que «La tecnología no se usa en todas las materias con igual intensidad y, por consiguiente, hay un porcentaje elevado de materias en las cuales el nivel de integración es prácticamente inexistente...». Respecto a nuestro sistema escolar, en un trabajo reciente de Barquín (2011, p. 36) se pone de manifiesto que el $86 \%$ del profesorado reconoce el valor didáctico de las nuevas herramientas, lo cual no es óbice para que el 34\% de ellos admita la escasa incidencia de estos medios en el aprendizaje escolar.

\section{«El rincón del ratón» desde la perspectiva de la TT}

Cada vez resulta más evidente que los medios tecnológicos provocan cambios significativos en la estructura y funcionamiento de las organizaciones. Por esta razón en los programas de TT, se dedica un esfuerzo importante a preparar la recepción. Se planifican distintas actuaciones con los miembros de la organización para asimilar los nuevos dispositivos tecnológicos incorporados a su quehacer laboral. Un trabajo que, de algún modo, pretende ayudar a resolver «la paradoja de la estabilidad y el cambio entre los miembros de la organización»(Lam, 2011, p. 174). En este sentido resulta de gran interés estudiar cómo se ha realizado el equipamiento en el proyecto «El rincón del ratón», pues el profesorado llega a invisibilizar los recursos TIC al conferirles «un carácter subsidiario y de apoyo de la acciones que se llevan a cabo en el contexto real del aula» (Ramírez y Martín, 
2011, p. 23). ¿Cómo ha asimilado el profesorado la incorporación del «pupitre informático» a su quehacer de aula?

Si tomamos el modelo de Rubiralta (2004) para el análisis de la TT, hemos de prestar atención a la estructura sobre la que se sustenta la implementación de estos programas. Estructura que se configura, según otros autores, por diferentes instancias, alguna de las cuales ejerce la función de facilitadora, de intermediación entre el ámbito de la innovación tecnológica y el ámbito de recepción de aquélla. Papel desempeñado, en el caso del proyecto «El rincón del ratón», por la Fundación Sánchez Ruipérez que, a través del Centro Internacional de Tecnologías Avanzadas para el Medio Rural (CITA) ${ }^{3}$, coordina la implantación en su entorno inmediato del proyecto. La estructura se fortalece al contar con IBM como socio tecnológico, multinacional propietaria del pupitre para educación infantil KidSmar ${ }^{4}$ que es la base de «El rincón del ratón». Por otra parte, el proyecto se desarrolla en un entorno rural con un nivel socioeconómico bajo, en virtud de lo cual recibe apoyo financiero de los fondos estructurales FEDER de la Unión Europea.

En segundo lugar, la estructura se consolida, según Rubiralta, activando instrumentos tales como contratos, cesión de licencias, movilidad del personal, servicios de apoyo, etc. Pues bien, en el caso que nos ocupa, cada una de las aulas de educación infantil de los 8 centros que participan en el proyecto fueron equipadas con un pupitre KidSmart. Además del equipo, se les ofreció software educativo, material didáctico, formación del profesorado, redes de intercambio de experiencias, licencias de usuarios y servicios de mantenimiento, etc ${ }^{5}$. Aunque no hay propiamente movilidad del personal, los centros del proyecto cuentan con el apoyo y asesoramiento de las «orientadoras tecnológicas especialistas en educación infantil» itinerantes del CITA, además de un coordinador TIC perteneciente al claustro (recordemos que estos centros podrían estar participando, a la vez, en el Plan Escuela 2.0 promovido por el Ministerio de Educación).

El tercer elemento del modelo de Rubiralta, es la participación o implicación de los diferentes actores. Por la naturaleza de las organizaciones que nos ocupan, esta es una dimensión fundamental, en la medida que se externalizan ciertas tareas y en su ejecución se implica a nuevos actores no siempre relacionados con la educación, ni vinculados al centro. Todo lo cual configura un complejo entramado de relaciones con nodos críticos respecto a la eficiencia. Los proyectos en tales circunstancias responderían al patrón del «modelo mediterráneo» de TT según Rubiralta (los otros modelos son el nórdico, el anglosajón y el centroeuropeo). En definitiva y siguiendo a Aceytuno y Cáceres (2011), el proyecto «El rincón del ratón» se configura como subsistema local (se concibe para aplicarse en una comarca de la provincia de Salamanca) y aprovecha sinergias, en ocasiones informales, como cuando se implica a la 
asociación de madres y padres — AMPA— o al ayuntamiento con ayudas económicas. El modelo mediterráneo se caracteriza no tanto por las innovaciones ni las patentes que genera sino por la proliferación de publicaciones, estudios, reuniones de carácter científico y formativo.

\section{CONSIDERACIONES DE ORDEN METODOLÓGICO}

En este trabajo nuestro objetivo se centra, prioritariamente, en analizar cómo los actores de una organización se apropian y asimilan la tecnología que se les facilita a partir de una iniciativa de innovación. Bajo el enfoque actual de la tecnología, el conocimiento es tanto o más importante que el artefacto material. Lo cual significa, entre otras cosas, que en los programas de TT actuales lo sustantivo sería el conocimiento (Pinto, 2012). De manera que estos programas alcanzarán los objetivos previstos, si el conocimiento asociado es «apropiado» principalmente por los actores y también por el resto de las instancias de la organización receptora (Molero, 2008; Segrestin, 2004).

A partir de este planteamiento conceptual, tomamos el proyecto de «El rincón del ratón» para dilucidar aquí cuestiones como las siguientes: ¿Qué caracteriza al citado proyecto de transferencia? ¿Cómo se prepararon los centros participantes para trabajar con los KidSmart? ¿Qué tipo de iniciativas de intermediación adopta el CITA y cómo las perciben los actores? ¿Se le da visibilidad al aprendizaje organizacional en la nueva situación?

En pos de estos interrogantes hemos retomado el proyecto inicial (ver Nota 1), de cuyos datos y materiales de campo se nutre el presente trabajo. Y para disponer de la perspectiva y claves con las que interpretar los resultados, explicitamos los elementos de orden metodológico más relevantes. La muestra la constituyen los 8 centros de Educación Infantil y Primaria que participan en el proyecto «El Rincón del ratón» promovido por el CITA para el segundo ciclo de Infantil. Del conjunto de centros uno es concertado y los siete restantes son públicos, característica distintiva que no se ha mostrado demasiado relevante para nuestros propósitos por lo que la hemos desestimado.

En el mencionado ciclo de Infantil había 17 aulas y otros tantos profesores en el conjunto de los centros estudiados. Con la totalidad de estos docentes se mantuvo una entrevista semiestructurada (media hora de duración y realizadas entre septiembre y noviembre de 2010), las cuales fueron grabadas y luego transcritas. Además a las familias con hijos en alguna de estas aulas, se les pasó una encuesta de 12 ítems de respuesta múltiple, la cual fue elaborada por el equipo de investigación y sometida a la revisión 
de tres jueces externos. A través de los alumnos se les hizo llegar a las familias un ejemplar de la encuesta en marzo de 2010, durante la segunda semana debían entregarla en el centro en sobre cerrado y anónimo. Así se recogieron 243 encuestas válidas que se aproximan a los dos tercios de la población total de familias. Para el tratamiento de los materiales y datos se han utilizado procedimientos eminentemente cualitativos, también a los estadígrafos descriptivos aplicados a la encuesta.

\section{EXPOSICIÓN Y COMENTARIO DE RESULTADOS}

Es en el último eslabón de la cadena, el centro, donde la TT llega a los usuarios finales, docentes y estudiantes, no sin escapar del todo de los condicionamientos económicos, políticos y de gestión de las empresas y administraciones implicadas. Aunque el proyecto «El rincón del Ratón» se focaliza en la Educación Infantil, acaba integrándose en el centro escolar, cuya organización debe «digerir» la nueva circunstancia. De hecho, algunos autores señalan a la organización receptora de la TT como factor crítico en el éxito de la misma; a la organización en su conjunto y no sólo a un servicio o departamento concreto (Lam, 2010). Razón por la que focalizamos la atención sobre los principales actores implicados en el proyecto y sobre los centros que lo aplican, en tanto que corresponsables de su éxito o fracaso. En cualquier caso, la experiencia de trabajar en las aulas con el KidSmart nos ofrece claves significativas sobre cómo se articula y acoge en la institución escolar un programa de $\mathrm{TT}^{6}$.

\section{El apoyo de las familias}

Es evidente que la familia es un agente activo en el centro, máxime en las primeras etapas escolares. No sólo porque es la instancia primaria de socialización de los escolares, sino también por la posibilidad de participar a través del Consejo Escolar o el AMPA en la toma de decisiones que revierten de manera más o menos directa en el funcionamiento general del centro. Por tanto, su cometido dentro del proceso de TT no es insustancial, la aceptación o no, la relevancia o no que otorguen a esa tecnología es, en gran medida, un generador de expectativas y exigencias trasladables a la educación formal de sus hijos y por ello al centro y al profesorado. Como se desprende de los datos recogidos en la Tabla 1, las familias $(\mathrm{N}=243)$ cuentan mayoritariamente en su hogar con tecnología similar a la que se encontrarán sus hijos en las aulas de Educación Infantil y complementada con otras tecnologías como TDT, móviles, videoconsolas, mp3, etc. Quizá no el KidSmart con que les dota el CITA, pero sí con los fundamentos tecnológicos con los que opera el pupitre. 
Tabla 1.

Equipamiento tecnológico de las familias participantes

\begin{tabular}{lccccccc}
\hline & \multicolumn{2}{c}{ TDT/DVD } & \multicolumn{2}{c}{ INTERNET } & ORDENADOR & \\
\hline & $\%$ NO & $\%$ SI & $\%$ NO & $\%$ SI & $\%$ NO & $\%$ SI & f \\
\hline Centro 1 & .0 & 100 & 55.6 & 44.4 & 55.6 & 44.4 & 19 \\
Centro 2 & .0 & 100 & 55 & 45 & 25 & 75 & 20 \\
Centro 3 & .0 & 100 & 40 & 60 & 10 & 90 & 10 \\
Centro 4 & .0 & 100 & 34.5 & 65.5 & 12.7 & 87.3 & 55 \\
Centro 5 & .0 & 100 & 51.9 & 48.1 & 19.2 & 80.8 & 53 \\
Centro 6 & .0 & 100 & 33.3 & 66.7 & 6.7 & 93.3 & 45 \\
Centro 7 & .0 & 100 & 100 & 0 & 50 & 50 & 4 \\
Centro 8 & .0 & 100 & 45.9 & 54.1 & 21.6 & 78.4 & 37 \\
Total & $\mathbf{0}$ & $\mathbf{1 0 0}$ & $\mathbf{4 3}$ & $\mathbf{5 7}$ & $\mathbf{1 7 . 4}$ & $\mathbf{8 2 . 6}$ & $\mathbf{2 4 3}$ \\
\hline
\end{tabular}

Tal como ponen de manifiesto otros estudios con muestras mucho más amplias (INE, 2012), el equipamiento de los hogares es similar y en ocasiones mejor que el de los centros escolares. Este grado de socialización de las familias en el uso de las tecnologías, explicaría sus respuestas a uno de los ítems de la encuesta inicial, cuando se les preguntaba: ¿Está de acuerdo con que se dedique tiempo al uso de las tecnologías en el ámbito escolar? Los padres se manifiestan mayoritariamente muy de acuerdo o de acuerdo, el $98,3 \%$, con la dedicación de tiempo en la escuela para el uso de tecnologías, un reducido $1,3 \%$ se muestra indiferente y un $0,4 \%$ está totalmente en desacuerdo (Gráfico 1).

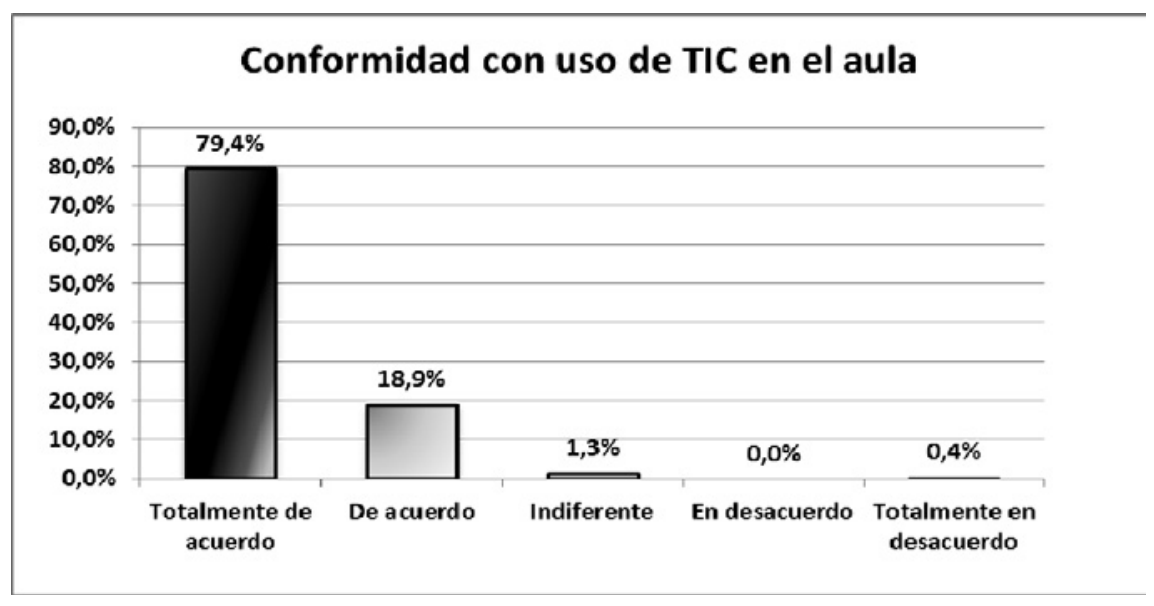

Gráfico 1. Conformidad/disconformidad con el uso de las TIC en las aulas. 
El perfil dibujado por el gráfico precedente no deja lugar a dudas sobre la convicción de las familias respecto al valor pedagógico de las TIC en las aulas, tal como también concluyen Ballesta y Cerezo (2011). Esta constatación invita a prestar atención a los datos de la Tabla 2 por cuanto muestran indicios sobre una posible brecha: la que se refiere a las prácticas de uso. Pese a que las tecnologías son tan iguales en forma (hardware) y tan versátiles en fondo (software), los usos que otorgan a las mismas en el seno familiar tienden al ocio $(94,2 \%)$, mundo laboral $(42,1 \%)$ e información $(53,3 \%)$ y apenas tocan el ámbito de la formación $(3,3 \%)$, como se puede apreciar en la tabla siguiente.

Tabla 2.

Los distintos usos familiares de los recursos tecnológicos en el hogar

\begin{tabular}{lccccccccc}
\hline & \multirow{2}{*}{ OCIO } & \multicolumn{2}{c}{ LABORAL } & \multicolumn{2}{c}{$\begin{array}{c}\text { INFORMA- } \\
\text { TIVO }\end{array}$} & \multicolumn{2}{c}{$\begin{array}{c}\text { FORMA- } \\
\text { CIÓN }\end{array}$} \\
\hline & $\%$ NO & $\%$ SI & $\%$ NO & $\%$ SI & $\%$ NO & $\%$ SI & $\%$ NO & $\%$ SI & f \\
\hline Centro 1 & .0 & 100 & 55.6 & 44.4 & 55.6 & 44.4 & 77.8 & 22.2 & 19 \\
Centro 2 & 20 & 80 & 70 & 30 & 45 & 55 & 80 & 20 & 20 \\
Centro 3 & 10 & 90 & 50 & 50 & 10 & 90 & 90 & 10 & 10 \\
Centro 4 & 5.5 & 94.5 & 43.6 & 56.4 & 41.8 & 58.2 & 72.7 & 28.3 & 55 \\
Centro 5 & 7.7 & 92.3 & 67.3 & 32.7 & 57.7 & 42.3 & 80.8 & 19.2 & 53 \\
Centro 6 & 2.2 & 97.8 & 53.3 & 46.7 & 42.2 & 57.8 & 68.9 & 31.1 & 45 \\
Centro 7 & 0 & 100 & 75 & 25 & 100 & 0 & 100 & .0 & 4 \\
Centro 8 & 2.7 & 97.3 & 64.9 & 35.1 & 51.4 & 48.6 & 75.7 & 24.3 & 37 \\
Total & $\mathbf{5 . 8}$ & $\mathbf{9 4 . 2}$ & $\mathbf{5 7 . 9}$ & $\mathbf{4 2 . 1}$ & $\mathbf{4 6 . 7}$ & $\mathbf{5 3 . 3}$ & $\mathbf{9 6 . 7}$ & $\mathbf{3 . 3}$ & $\mathbf{2 4 3}$ \\
\hline
\end{tabular}

Las respuestas de los padres apuntan claramente a que el uso que sus hijos hacen de las tecnologías en los respectivos domicilios, se centra mayoritariamente en el entretenimiento y muy poco en lo pedagógico, aunque confían en que la escuela asuma mayor protagonismo en este terreno. De modo que los niños se socializan y refuerzan un tipo de uso de las tecnologías en el medio familiar que no se corresponde con el que se les va a proponer y exigir en las aulas. ¿Esta falta de convergencia en los usos puede constituir una brecha que distancie un poco más a las escuelas de su entorno sociocultural? ¿Es factible didácticamente revertir esta deriva en el uso de los medios tecnológicos? En cualquier caso, el uso espontáneo de estos medios se orienta hacia el entretenimiento, abundando en el «capitalismo de ficción» del que habla Verdú (2003). 


\section{El centro y las aulas de Educación Infantil}

Los centros educativos, entendidos como la organización receptora de la TT, deberían ocupar un papel fundamental y estratégico en dicho proceso. Las dotaciones de equipos tecnológicos (Pizarras digitales, ordenadores, cañones, pupitres digitales, etc.) son muy diversas y varían según los centros (Tabla 3). Por ejemplo, si aludimos al equipamiento informático nos encontramos con un total de 167 ordenadores, cuya distribución varía tanto intercentros como intracentro al tener que repartirse por aulas y ciclos. Las diferencias se hacen más llamativas, si cabe, cuando se considera la dotación de un «intangible» como es la conectividad a la red. Dado el entorno socioeconómico de los ocho centros, ¿disponen de un buen acceso a la red para operar con los medios disponibles?

Tabla 3.

Número de ordenadores, PDI y acceso a Internet en los espacios del centro

\begin{tabular}{|c|c|c|c|c|c|c|c|c|c|c|c|c|c|c|c|c|c|c|c|c|}
\hline & \multicolumn{3}{|c|}{$\begin{array}{c}\text { Aula de } \\
\text { informática }\end{array}$} & \multicolumn{3}{|c|}{$\begin{array}{l}\text { Aula de } \\
\text { Infantil }\end{array}$} & \multicolumn{3}{|c|}{$\begin{array}{c}\text { Aula de } \\
\text { primaria }\end{array}$} & \multicolumn{3}{|c|}{$\begin{array}{l}\text { Sala de } \\
\text { profesor }\end{array}$} & \multicolumn{3}{|c|}{$\begin{array}{l}\text { Aula de } \\
\text { tecnología }\end{array}$} & \multicolumn{3}{|c|}{$\begin{array}{c}\text { Otros } \\
\text { espacios }\end{array}$} & \multicolumn{2}{|c|}{ Total } \\
\hline Centro & 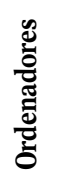 & 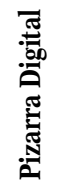 & 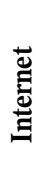 & 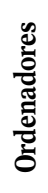 & 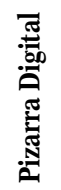 & 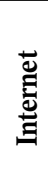 & 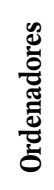 & 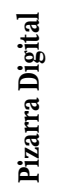 & 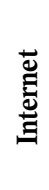 & 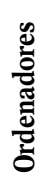 & 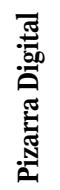 & 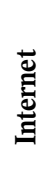 & 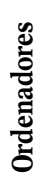 & 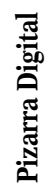 & 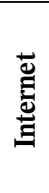 & 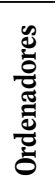 & 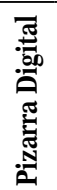 & 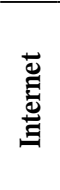 & 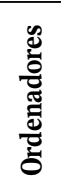 & 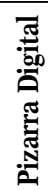 \\
\hline Centro 1 & 8 & 1 & Sí & 1 & 0 & Sí & 6 & 0 & Sí & 4 & 0 & Sí & 0 & 0 & No & 0 & 1 & No & 19 & 2 \\
\hline Centro 2 & 12 & 0 & Sí & 0 & 0 & - & 4 & 2 & - & 0 & 0 & Sí & 0 & 1 & Sí & 3 & 0 & Sí & 19 & 3 \\
\hline Centro 3 & - & 1 & Sí & 1 & 0 & No & 0 & 0 & No & 1 & 0 & Sí & 0 & 0 & No & 0 & 0 & No & 2 & 1 \\
\hline Centro 4 & 26 & 1 & Sí & 3 & 0 & - & 2 & 2 & - & 2 & 0 & Sí & 0 & 0 & Sí & 0 & 0 & Sí & 40 & 10 \\
\hline Centro 5 & 10 & 1 & Sí & 3 & 1 & Sí & 6 & 4 & Sí & 0 & 0 & No & 1 & 1 & Sí & 4 & 0 & Sí & 24 & 7 \\
\hline Centro 6 & 23 & 2 & Sí & 3 & 0 & - & 5 & 5 & - & 1 & 0 & Sí & 0 & 0 & Sí & 5 & 0 & Sí & 37 & 7 \\
\hline Centro 7 & - & 1 & Sí & 1 & 0 & No & 0 & 0 & No & 1 & 0 & Sí & 0 & 0 & No & 0 & 0 & No & 2 & 1 \\
\hline Centro 8 & 17 & 0 & Sí & 3 & 0 & Sí & 3 & 2 & Sí & 0 & 0 & No & 0 & 0 & No & 1 & 1 & Sí & 24 & 3 \\
\hline Total & 96 & 7 & - & 15 & 1 & - & 26 & 15 & - & 9 & 0 & - & 1 & 2 & - & 13 & 2 & - & 167 & 27 \\
\hline
\end{tabular}

Según los datos que nos aportan los centros estudiados, la totalidad de ellos disponen de material didáctico en soporte digital (software educativo), obtenido a través de distintas fuentes, según se aprecia en el Gráfico 2. Hay diferentes agentes o instituciones que proporcionan dichos materiales, pero cabe destacar dos fuentes coincidentes de obtención de aquéllos: por un lado, las editoriales $(87,5 \%)$ y, por otro, Internet $(100 \%)$. En contraposición, ninguno de los centros obtiene el material a través de empresas especializadas (Zetamultimedia, Anaya Multimedia, Microsoft, etc.) y tampoco demasiados de instituciones como el CFIE $^{7}$ o el MEC (25\%). 


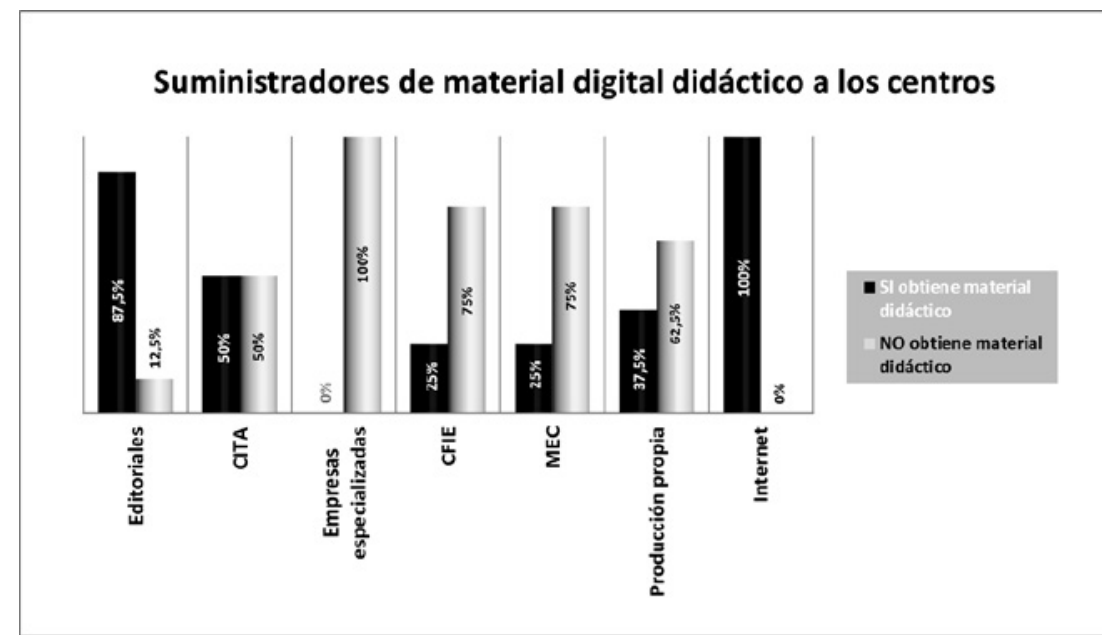

Gráfico 2. Proveedores de material didáctico en soporte digital

Otro dato importante que obtenemos de los centros es el que hace referencia a los apoyos, el mantenimiento y asesoramiento técnico de los recursos tecnológicos (Gráfico 3) donde nos encontramos con una diversidad de servicios. Según la información recogida prevalece el perfil de un docente que hace la función y las tareas inherentes a la coordinación TIC, para lo que la Administración les reconoce 2 horas lectivas a la semana por centro. Pero también existen otras opciones e incluso la combinación de varios servicios de asesoramiento y mantenimiento técnico en un mismo centro, según los proyectos en los que participa.

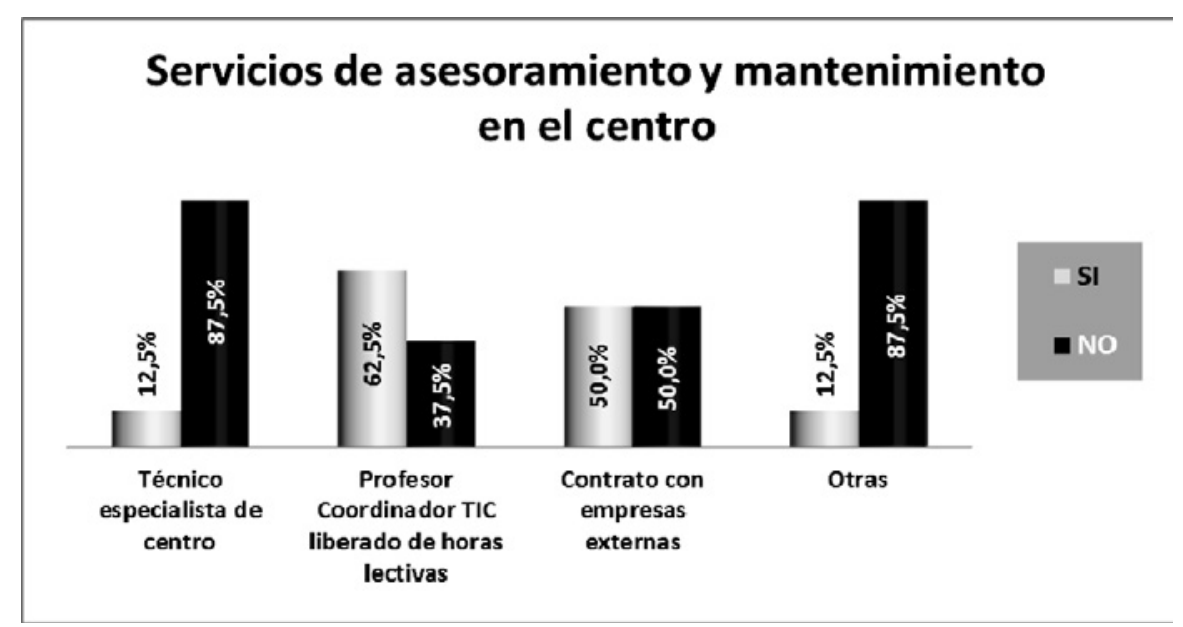

Gráfico 3. Apoyo y asesoramiento tecnológico 
Si nos centramos en la etapa de Educación Infantil, objetivo de la TT del proyecto de «El Rincón de Ratón», los datos recogidos ponen de manifiesto que las aulas de esta etapa ya estaban dotadas de ordenadores antes de la llegada de los pupitres KidSmart de IBM. De hecho, el 83,33\% de las aulas de infantil ya contaban con un ordenador y únicamente el $16,67 \%$ no lo tenían. Por otro lado, se observa que el patrón de distribución interna de los recursos tecnológicos manifiesta una clara asimetría, como mínimo, entre las etapas en un mismo centro (Tabla 3). Es llamativo el caso, por ejemplo, de la Pizarra Digital Interactiva (PDI) que, de las 17 aulas de infantil participantes en la investigación, tan sólo una de ellas está dotada con PDI. ¿El desequilibrio se debe a las diferencias en el «estilo docente» entre infantil y primaria o a la versatilidad de uso de los artefactos tecnológicos?

En cualquier caso habrá que seguir indagando en las razones que explican dicha asimetría, por qué se producen tales desequilibrios internos. El hecho remite, sin duda, a cómo las instancias organizativas de los centros establecen prioridades en la distribución de los recursos, a cómo se negocian los criterios de asignación e incluso si prevalece lo «técnico» sobre lo «democrático». En el caso de otras dotaciones, como el de la conectividad, dependen de los balances económicos de las operadoras y de sus contratos con la Administración educativa o de cómo se gestione desde la dirección el presupuesto disponible. En definitiva, cuestiones sobre las que habremos de seguir profundizando.

Una última observación: los centros tienen, además del pupitre informático, una aceptable dotación de materiales y equipos tecnológicos. Con todo ello, nos preguntamos, ¿qué ha aprendido la organización? Cuando la tecnología transferida es asimilada y apropiada por los actores, entonces se consolidan los cambios generados pudiendo afirmar que la organización ha aprendido (Lam, 2011, p. 169). Y cuando esto se produce, entonces se muestran y recogen en los reglamentos y otros documentos de la organización. En nuestra muestra sólo hemos encontrado un centro - el concertado- que en su web nos habla del proyecto y remite a documentos del CITA. En el resto no hemos encontrado ninguna referencia, directa o indirecta, a su participación en el proyecto «El Rincón del ratón». Eso sí, uno de éstos exhibía el certificado de la Administración reconociendo la excelencia en la aplicación de las TIC. Desde luego, sería de interés indagar en las razones aducidas por los agentes de la organización sobre la detectada invisibilidad del proyecto en su web.

\section{La intermediación tecnológica del CITA}

El CITA, continuando con el modelo, constituye la estructura de intermediación que asume la responsabilidad del diseño e implementación del 
proyecto tecnológico «El Rincón del Ratón». Son los encargados de contactar con los centros, instalar los pupitres KidSmart en las aulas, así como del mantenimiento de los mismos. Hasta aquí costaría encontrar diferencias entre una empresa informática y el CITA, puesto que todos estos servicios pueden ser plenamente gestionados por cualquiera de las dos.

El matiz diferenciador del proceso de TT que estudiamos, radica en el protagonismo asumido por el CITA en la implementación. No se limita únicamente a la dotación de equipamiento, proporciona distintos apoyos con los que trata de facilitar la integración de la tecnología en el contexto particular para la que va destinada, en particular la Educación Infantil. Para ello no sólo se incorpora software educativo (aportado por IBM en tanto que socio tecnológico) sino que a su vez adquiere una importancia crucial la figura del asesor por cuanto participa en la implantación del proyecto «El rincón del ratón» en las aulas.

El asesor es una persona con formación pedagógica, conocimientos informáticos y sobre los materiales educativos instalados en el pupitre KidSmart. Su función principal es la de apoyar a los docentes con una hora quincenal en la integración de los pupitres en las actividades de aula, trabajando con los alumnos para que adquieran competencias digitales básicas y puedan manejar adecuadamente el equipamiento disponible. Además asesora a los docentes en el uso de posibles herramientas TIC y materiales según las necesidades didácticas. Todo ello sustentado con la organización de actividades como congresos, seminarios o promocionando investigaciones como la que sirve de base a este texto. Razón por la que, como señala Molero (2008, p. 641), puede considerarse un facilitador de servicios intensivos en conocimientos.

Por otra parte, cabe destacar que el CITA ofrece a los docentes, fuera del proyecto «El Rincón del Ratón», diversos servicios complementarios. Un ejemplo de ello son los cursos sobre el uso de determinadas herramientas como puede ser la PDI, elaboración de objetos de aprendizaje, etc. Eso sí, estos servicios extra conllevan un coste económico para los docentes. No obstante, a lo largo del año escolar el profesorado de esta comarca, tiene oferta de numerosos cursos de formación subvencionados, en su mayoría, por la Administración educativa.

\section{Los docentes como actores de la recepción}

No cabe duda que gran parte de la responsabilidad en la aplicación de los recursos TIC en el aula recae sobre el profesorado. En el caso que nos ocupa son, en última instancia, los docentes de Educación Infantil de cada 
centro, quienes deciden usar o no la tecnología disponible y de qué modo utilizarla en el desarrollo de sus clases. La decisión no es estrictamente técnica sino que intervienen variables de muy distinta naturaleza y, desde luego, algunas escapan al ámbito competencial del docente de aula. A través de las entrevistas semiestructuradas realizadas en las visitas a los centros, se pueden esbozar las actitudes que mostraban los 17 profesores ante la inminente incorporación de sus aulas al proyecto «El Rincón del Ratón».

Prima mayoritariamente una actitud positiva ante la integración de las TIC, tal como se desprende de las respuestas dadas a la siguiente pregunta: ¿Qué motivos te han animado a participar en el proyecto "El Rincón del Ratón»? Los docentes indican diversos factores que animaron su incorporación al proyecto, entre ellos destacan los que aluden a la motivación que despiertan estos medios entre los niños, lo familiarizados que están los alumnos con las tecnologías, contar con la ayuda de un asesor o el prestigio que el CITA tiene en la comarca son algunos de los más relevantes para ellos. Parte de estas motivaciones se explicitan en las siguientes respuestas:

Profesor 1: "Cuando me lo presentaron me pareció un proyecto muy atractivo para los niños. A ellos les resulta muy motivador y a la vez que están jugando están aprendiendo».

Profesor 12: "Uso e introducción del ordenador como un rincón más de la actividad del aula».

Profesor 16: «Disponer de un monitorla que colabore».

Profesor 3: "El tener en el aula un ordenador a disposición y poderlo utilizar los niños. También es muy interesante el apoyo de la monitora».

Uno de los principales problemas a resolver en los programas de TT es la formación previa que tiene el profesorado para el uso de los recursos dentro del aula, por cuanto esta formación les debería permitir asimilar lo novedoso de la tecnología recibida. Razón por la cual se les planteó la siguiente cuestión: ¿Cómo valoras tu formación previa en TIC, independientemente de El Rincón del Ratón? En términos generales destaca su actitud positiva, aunque cauta, ante la formación, la mayoría reconoce haber participado en cursos a través del CFIES o el CITA para el uso de las TIC.

Profesor 7: "He recibido cursos por parte de CFIE y del CITA, sobre todo de carácter didáctico, pero tengo que reconocer que mi formación está todavía en fase inicial». 
Profesor 8: "Muy positiva, por cursos de formación permanente del profesorado y por mi trabajo personal».

Profesor 13: "Mi formación en TIC es media. He recibido cursos en CFIES, CITA,...».

Las coincidencias en sus valoraciones se disipan cuando se analiza el contenido de las respuestas a preguntas que no dependen tanto del posicionamiento personal sobre su propia actividad, sino que se les invita a valorar a otros agentes participantes en el proceso de TT. En tal dirección va la siguiente pregunta: ¿Cómo ha sido la información que has recibido para la integración de este recurso en las aulas? Cuestión concreta sobre la que encontramos diversidad de respuestas. Están, por un lado, quienes ponen el énfasis en los aspectos que consideran más o menos negativos:

Profesor 1: "Escasa, ya que me presentaron el proyecto, (ien qué consistía?), pusieron el ordenador pero ni siquiera lo encendieron. "Ahí está, haz lo que quieras", sin explicaciones de los programas que contenia».

\section{Profesor 17: «Poca...»}

Y en otros casos con valoración positiva, como apuntan las siguientes respuestas:

Profesor 10: "Adecuada. Satisfactoria. Desde el CITA se nos facilitó la organización del proyecto, sus aplicaciones didácticas, el software necesario, los recursos informáticos y el asesoramiento continuado».

Profesor 4: «Ha sido correcta, dando la oportunidad de plantear las dudas que podian surgir».

Si miramos en conjunto las respuestas de los 17 profesores, observamos que predominan las que muestran una actitud neutral (6 profesores) e incluso negativa (7 profesores). Por otro lado están quienes manifiestan una actitud más favorable a la información recibida (4 profesores). Respecto a las soluciones demandadas en el plano técnico por el recurso, el profesorado tiene claro que el CITA o el centro les prestarán apoyo para superar las dificultades. Cuestión a la que se dirige la siguiente pregunta: ¿Existen medidas de apoyo en caso de problemas técnicos con el Rincón del Ratón?

Profesor 13: "Intentamos resolver los problemas a nivel de centro. El CITA nos ha ayudado puntualmente cuando lo hemos necesitado». 
ÁNGEL SAN MARTÍN ALONSO, JORGE MARTÍN DOMÍNGUEZ Y ELENA RAMÍREZ ORELLANA RECEPCIÓN Y ASIMILACIÓN DE LAS TECNOLOGÍAS EN CENTROS ESCOLARES: EL PROYECTO «EL RINCÓN DEL RATÓN»

Profesor 7: "Hasta ahora no se han planteado, pero sé que del CITA acudirian en cuanto surgieran».

Nos encontramos así que las apreciaciones manifestadas por el profesorado se convierten en críticas para el éxito del proceso de TT en cuanto al uso de las tecnologías en las aulas. Se desprende que en tales decisiones la "cuestión didáctica», como alcanzar los objetivos educativos, no tiene tanto peso como otros factores asociados al prestigio, a las expectativas profesionales e incluso a consideraciones de orden moral y no ya sólo a prejuicios conservadores como a veces se esgrime. Quizá por ello se podría afirmar, como apunta Sigmund (2012), que la relación de los usuarios con las tecnologías - por ejemplo con Google o e-Bay_ pueda explicarse mejor según el modelo del «dilema del prisionero» basado en el prestigio y confianza que el medio le merece al usuario. Puede que este modelo resulte demasiado aleatorio, pero lo cierto es que el nivel de conocimiento de la herramienta no es proporcional al grado de uso de aquélla en las aulas, y el proyecto que nos ocupa tampoco escapa a esta tendencia.

\section{CONSIDERACIONES FINALES}

A partir de los interrogantes planteados en el segundo epígrafe, concluimos, en primer lugar, que «El Rincón del Ratón» sigue básicamente el protocolo de transferencia central-periférico, por cuanto se concibe para su diseminación en una instancia superior (el CITA) y luego se invita a los centros a participar. Aparte de dotar a las aulas de infantil de diverso material, el principal apoyo se focaliza en actuaciones puntuales sobre la formación del profesorado y en mucha menor medida sobre el conjunto del centro escolar. El modelo no deja demasiado espacio a la reciprocidad, a darles voz a padres, profesorado y equipos directivos sobre las circunstancias de la implementación del programa de TT. Como tampoco es muy permeable a la coordinación con otras iniciativas semejantes que puedan estar llegando al centro, por ejemplo el ministerial Escuela 2.0 o las específicas de la Junta de Castilla y León. ¿Cómo puede asimilar la organización la pluralidad de estímulos de proyectos diferentes?

En segundo lugar destacamos que hay algo más que la mera distribución de artefactos como el KidSmart. Hay acciones de intermediación como la formación, asesoramiento e investigación que pone en valor el conocimiento inherente a la tecnología transferida. De todos modos, pese a ser bien acogido el proyecto por los actores de la organización (familias y profesorado manifiestan tener mucha confianza en las tecnologías), luego no se visibilizan los aprendizajes desarrollados en la integración de la dotación de las tecnologías recibidas. Resulta revelador que en la web de los centros 
estudiados no se recoja de algún modo su participación en el proyecto del CITA, como tampoco se deja constancia en el proyecto educativo. Sí se refleja, en cambio, en el reglamento con la finalidad de regular el acceso y la conservación de los equipos. Cabe así preguntarse, ¿se está, como señala Cuban (2011), haciendo un uso considerable de las TIC pero sin generar cambios significativos en la enseñanza? ¿Cómo se flexibilizan los patrones organizativos que regulan las actividades de aula?

La literatura más reciente insiste en la función determinante del contexto organizativo para el éxito de las tecnologías transferidas (Jiménez, 2003). Es obvio que en el programa que nos ocupa los avances experimentados en esta dimensión son escasos, tal vez porque el programa se centra en la etapa de Educación Infantil. Etapa que, al gozar de cierta «autonomía» en el funcionamiento ordinario, no logra trasladar sus innovaciones al conjunto de la organización del centro. Salvo la que hace a ésta, como irónicamente señala Lanier (2011b, p. 125), más dependiente de los «servicios técnicos». Aunque sea para uso exclusivo de infantil, el KidSmart introduce en los centros nuevos actores, funciones y dependencias que de algún modo deberían ser abordadas. Al no hacerlo se alimentan dilemas e indefiniciones en la trama organizativa de estos centros: ¿Los niños aprenden más o menos con el nuevo artefacto? ¿Qué responsabilidad puede asumir el técnico/asesor de una empresa sobre la marcha del aula de una escuela pública?

Por último y con carácter prospectivo, habría que seguir indagando por qué estos programas transfieren de forma intensiva los artefactos y en menor grado el know-how asociado. Hecho que ratifica la observación de Zeichner (2010, p. 160) cuando afirma: «Los mundos de la investigación del profesor y de la investigación académica raramente se entrecruzan». Es muy significativo que los numerosos estudios vinculados al proyecto KidSmart no hayan sido material de trabajo para el profesorado de estos ocho centros que aplican dicha tecnología. ¿El manejar estos conocimientos contribuiría a superar los numerosos dilemas que le surgen a los docentes al decidir el incorporar o no a sus tareas los medios tecnológicos? Desde luego, no se nos oculta que esas decisiones están entreveradas con las relaciones instituidas en las organizaciones receptoras y los intereses de quienes promueven la TT. Extremo que, sin duda, habrá que seguir estudiando a fin de optimizar las condiciones de recepción.

\section{NOTAS}

1 Este trabajo ha sido realizado gracias a la financiación del Ministerio de Ciencia e Innovación a través del proyecto de I+D (n. ${ }^{\circ}$ de referencia EDU2009-11295), así como por el apoyo recibido desde el Centro Internacional en Tecnologías Avanzadas (CITA) de la Fundación Germán Sánchez Ruipérez (http://www.citafgsr.org/cita). 
2 Estrategia Estatal de Innovación (e2i), 2010. Ministerio de Ciencia e Innovación. Disponible en: http://www.micinn.es/ [Consulta: 2012, 27 de marzo].

3 En la web oficial se recoge como uno de los objetivos del CITA el «Aplicar las Nuevas Tecnologías a la educación y la formación, haciendo especial énfasis en la teleformación.» Disponible en: http://www.citafgsr.org/cita/

4 Bajo el eslogan «Early learning program», IBM pone en marcha el programa KidSmart en 1999 donando un pupitre informático para educación infantil. Además se contempla tanto la formación del profesorado como la dotación de material didáctico e informático adaptado los requerimientos de esta etapa educación. Este programa está presente ya en más de 7.500 centros escolares de 60 países y en España participan unos 500 centros repartidos por todas las comunidades autónomas (web oficial: http://www.kidsmartearlylearning.org). También en esta web se ofrecen informes sobre las experiencias educativas internacionales, como el coordinado por Berry (2009).

5 Las ayudas y materiales de carácter pedagógico para el profesorado pueden encontrarse en: http://www.tecnologiaenlaeducacion.com/

6 En el Proyecto de Educación Infantil El rincón del ratón se establece un procedimiento de preparación de los receptores del KidSmart (pág. 21-23). Disponible en: http://cita.fundaciongsr.com/194/El-rincon-del-raton.

7 Las siglas aluden a los antiguos centros de profesores y que ahora en Castilla y León se denominan Centro de Formación e Innovación Educativa (CFIE). 


\section{REFERENCIAS BIBLIOGRÁFICAS}

Aceytuno, M. T., y Cáceres, F. R. (2011). Los modelos europeos de transferencia de tecnología universidad-empresa. [Ponencia] XIII Reunión de Economía Mundial.

Ballesta, J., y Cerezo, M. C. (2011). Familia y escuela ante la incorporación de las tecnologías de la información y la comunicación. Educación XX1, 14(2), 133-156.

Barquín, J., Ortiz, A., y Sepúlveda, M. P. (2011). Los datos de las TIC en los centros. En M. Sola, y J. F. Murillo, (Coords.), Las TIC en la Educación. Realidad y expectativas. Informe anual 2011 (pp. 7-45). Madrid: Fundación Telefónica-Ariel.

Bélanger, J., y Thuderoz, Ch. (2010). Le répertoire de l'opposition au travail. Revue Française de Sociologie. 51(3), 427-460.

Berry, C. (2009). Programa de Educación Infantil KidSmart de IBM. Recuperado de http://www.kidsmartearlylearning. org.

Castells, M. (2001). La galaxia Internet. Madrid: Areté.

COTEC (2011). Informe COTEC 2011. Tecnología e innovación en España. Madrid: COTEC.

Cuban, L. (2011). Entrevista. Cuadernos de pedagogía, 411, 40-45.

EACEA-Eurydice, (2011). Cifras clave sobre el uso de las TIC para el aprendizaje y la innovación en los centros escolares de Europa 2011. Brusselas: EACEA/Eurydice. Recuperado de http://goo.gl/3aektC

INE (2012). Encuesta sobre equipamiento y uso de tecnologías de la información y comunicación en los hogares 2012. Recuperado de http://goo.gl/Yn0DQy
Jiménez, J. M. (2003). Aspectos de la eficiencia en la transferencia de tecnología. Rev. de Investigación en Gestión de la Innovación y Tecnología, 14, 1-15.

Lam, A. (2011). Organizaciones innovadoras: estructura, aprendizaje y adaptación. Recuperado de http://goo.gl/ Z0B0oX

Lanier, J. (2011a). Personas desaparecidas. Claves de razón práctica, 218, 68-74.

Lanier, J. (2011b). Contra el rebaño digital. Barcelona: Debate.

Liu, L., Cleborne, M. \& LaMont, J. (2008). Assessment of Integration of Technology in Education: Countering the «No Significant Differences» Argument. Computers in Schools, 25(1/2), 1-9.

Molero, J. (2008). La transferencia de tecnología revisitada: conceptos básicos y nuevas reflexiones a partir de un modelo de gestión de excelencia. Arbor. Ciencia, Pensamiento y Cultura, 732, 637-651.

Mominó, J. M., Sigalés, C., Meneses, J. (2008). La escuela en la sociedad red. Internet en la educación primaria y secundaria. Barcelona: Ariel.

Onken, M., Fisher, C. \& Li, J. (2005). Perceived Impacts of Government Regulations on Technology Transfers. Journal of Nonprofit \& Public Sector Marketing, 13(1/2), 35-55.

Pedró, F. (2011). Tecnología y escuela: lo que funciona y por qué. Documento básico. XXVI Semana Monográfica de la Educación. Recuperado de http:// www.fundacionsantillana.com/.

Pinto, H. (2012). Instituciones, innovación y transferencia de conocimiento: contribuciones de los estudios sobre las variedades de capitalismo. Arbor. Ciencia, Pensamiento y Cultura, 188 (753), 31-47. 
Ramírez, E., y Martín, J. (2011). Enfoques metodológicos y prácticas con TIC. Un estudio en la etapa de educación infantil. En Peirats y San Martín (Coords.), Tecnologías educativas 2.0. Didáctica de los contenidos digitales (pp. 7-25). Madrid: Pearson.

Rubiralta, M. (2004). Transferencia a las empresas de la investigación universitaria. Descripción de los modelos europeos. Madrid: Fundación COTEC.
Segrestin, D. (2004). Les chantiers du manager. Paris: Armand Colin.

Sigmund, K. (2012). Reciprocidad indirecta, instinto de evaluación y prestigio. En J. Brockman, (Ed.), Cultura (pp. 117-129). Barcelona: Crítica.

Verdú, V. (2003). El estilo del mundo. Barcelona: Anagrama.

Zeichner, K. M. (2010). La formación del profesorado y la lucha por la justicia social. Madrid: Morata. 


\section{PERFIL ACADÉMICO Y PROFESIONAL DE LOS AUTORES}

Jorge Martín Domínguez. Profesor Ayudante Doctor en el Dpto. de Didáctica, Organización y Métodos de Investigación en la Universidad de Salamanca, realiza su actividad docente e investigadora de doctorado en el ámbito de la tecnología educativa.

Elena Ramírez Orellana. Profesora titular de Universidad del Dpto. de Didáctica, Organización y Métodos de Investigación en la Universidad de Salamanca, realiza su actividad docente e investigadora en el ámbito de tecnología educativa, actualmente es la IP de un proyecto de $\mathrm{I}+\mathrm{D}+\mathrm{i}$ realizado en colaboración con el CITA de la Fundación Sánchez Ruipérez.

Ángel San Martín Alonso. Profesor Catedrático de Universidad del Dpto. de Didáctica y Organización Escolar en la Universitat de València, realiza su actividad docente e investigadora en el ámbito de la organización escolar y la tecnología educativa, actualmente coordina el grupo de investigación CRIE.

Dirección de los autores: Ángel San Martín Alonso

Dpto. Didáctica y Organización Escolar

Universitat de València

Avda. Blasco Ibáñez, 30

46010 Valencia

E-mail: asanmart@uv.es

Elena Ramírez Orellana

Dpto. de Didáctica, Organización

y Métodos de Investigación

Universidad de Salamanca

P. $^{\circ}$ Canalejas, 169

37007 Salamanca

E-mail: ero@usal.es

Jorge Martín Domínguez

Dpto. de Didáctica, Organización

y Métodos de Investigación

Universidad de Salamanca

P. $^{\circ}$ Canalejas, 169

37007 Salamanca

E-mail: jorgemd@usal.es

Fecha Recepción del Artículo: 6. Enero. 2013

Fecha Modificación Artículo: 18. Junio. 2013

Fecha Aceptación del Artículo: 28. Agosto. 2013

Fecha Revisión para publicación: 15. Febrero. 2016 